\title{
Modeling the healing of damage of metal by high-energy pulsed electromagnetic field
}

\author{
K. V. Kukudzhanov ${ }^{\dagger}$ \\ ${ }^{\dagger}$ kconstantin@mail.ru
}

Ishlinsky Institute for Problems in Mechanics of RAS, 101b1, prosp. Vernadskogo, Moscow, 119526, Russia

The processes transformation of defects such as the intergranular micro-cracks are investigated. These processes occur in the material under the processing of metal samples with high-energy pulsed electromagnetic field inducing in the material a short pulse of high density electrical current. Investigation on basis of numerical coupled model of the impact of high-energy electromagnetic field on the pre-damaged thermal elasticplastic material with defects are carried out. The model accounts for melting and evaporation of metal and dependence of its physical and mechanical properties on temperature. Equations is solved numerically by finite elements method with adaptive mesh using on base of alternative Euler-Lagrange's method. Calculations has shown, that welding of crack and healing of micro-defects under treatment of short pulse of current are took place. Healing occurs by simultaneous reduction in length, the ejection of the molten metal into crack and closing of micro-crack shores. For macroscopic description of the healing process the material healed and damage parameters are entered. Healing of microcracks leads to the reduction of damage of the material. Shapes of microcracks practically does not affect on dependences of healed and damage from time under treatment of current pulse. These changes are affected by value of initial damage and initial length microcrack only. The dependences of healed and the damage from the time will be practically same for all different shapes of microdefects, provided that initial lengths microcracks and initial damages are equal for these different shapes of defects. Simple approximate piecewise-linear dependences of damage and healed from time and the initial damage are obtained.

Keywords: healing, micro-defects, electroplasticity, electropulsing, cracks arrest.

УДК 539.3

\section{Моделирование залечивания поврежденности металла}

\section{высокоэнергетическим импулысным электромагнитным полем}

\author{
Кукуджанов К. В. ${ }^{\dagger}$ \\ Института проблем механики им. А. Ю. Ишлинского РАН, пр-кт Вернадского, 101 - 1 Москва, 119526,, Россия
}

\begin{abstract}
Исследуются процессы трансформации дефектов типа межзеренных микротрещин, протекающие в материале при обработке металлических образцов импульсным высокоэнергетическим электромагнитным полем. Исследование осуществляется численно на основе связанной модели воздействия полем на предварительно поврежденный термоупругопластический материал с дефектами, которая учитывает плавление и испарение металла, а также зависимость всех его физико-механических свойств от температуры. Решение получающейся системы уравнений проводится методом конечных элементов на подвижных сетках с использованием смешанного эйлера-лагранжева метода. Моделирование показало, что одновременное уменьшение длины, выброса расплавленного металла внутрь трещины и смыкание берегов приводит к тому, что берега трещины начинают контактировать со струей расплавленного материала, и в результате этих процессов струя оказывается полностью зажатой берегами трещины. Таким образом, под действием импульсов тока происходит сварка берегов трещины и залечивание микродефектов. Для описания процессов залечивания вводятся параметры залеченности и поврежденности материала. Изучаются процессы изменения залеченности и поврежденности от времени при воздействии полем. Рассматриваются вопросы о выборе предпочтительных областей интегрирования и условий на их границах при моделировании этих процессов. Исследуется влияние формы микротрещин на изменение залеченности и поврежденности от времени. Залечивание микротрещин приводит уменьшению поврежденности материала. Форма микротрещин, практически не влияет на изменения залеченности и поврежденности во времени при воздействии на материал током. На эти изменения влияют лишь величина начальной поврежденности материала и начальная длина микротрещины. Зависимости залеченности и поврежденности материала во времени практически одинаковы для любых различающихся форм микротрещин при условии, что начальные длины и начальные поврежденности для этих различных форм дефектов равны. Получены простые приближенные кусочно-линейные зависимости изменения поврежденности и залеченности от времени и начальной поврежденности.
\end{abstract}

Ключевые слова: залечивание, микродефекты, электропластичность, электроимпульсный метод, торможение трещин. 


\section{1. Введение}

Микродефекты с линейными размерами порядка 10мкм являются наиболее распространенными в поликристаллическом металле. Их формируют, в первую очередь, поверхности соседних монокристаллов (зерен). Подобные дефекты всегда в каком-то количестве образуются между зернами после отливки, а также могут возникать и развиваться в металле в процессе его пластического деформирования. Наиболее опасными из микродефектов с точки зрения последующего макроразрушения изделия являются плоские микротрещины.

Гипотеза о том, что в проводящем материале может происходить залечивание (изменение) дефектов под действием кратковременных импульсов высокоэнергетического электромагнитного поля (далее - «ВЭМП») высказывалась рядом авторов [1-5] при объяснении явления электропластичности металлов. При этом в данных работах под залечиванием понималось как возникновение сжимающих напряжений в вершинах трещин, так и сближение их берегов, сопровождаемое выплавлением кратеров (пор) в вершинах микротрещин. Это создавало препятствия для дальнейшего развития трещин в материале.

В настоящее время гипотезу о залечивании дефектов в материале стало возможным перенести в разряд экспериментально наблюдаемого явления [6-10]. При этом данные эксперименты свидетельствуют о том, что происходит не просто возникновение сжимающих напряжений с выплавлением кратеров (пор) в вершинах, а изменение самой формы дефекта и восстановление сплошности структуры материала, сопровождаемые изменением объемного содержания микродефектов (вплоть до полного исчезновения некоторых микродефектов). Это приводит к уменьшению поврежденности материала и увеличению предельной пластической деформации до разрушения. Однако предлагаемые до настоящего времени математические модели [3-5,11-12] не позволяли объяснить эти экспериментальные факты. Естественно, что в таких условиях, вопросы об изменениях поврежденности и, тем более, закономерностях этих изменений при воздействии на материал ВЭМП оставались неизученными.

Для исследования физических процессов, происходящих в окрестности микротрещин под действием интенсивным импульсов тока и объяснения эффекта залечивания в работе [13] была предложена модель воздействия импульсного ВЭМП на предварительно поврежденный материал с дефектами, с помощью которой удалось математически описать экспериментально наблюдаемый процесс трансформации микродефектов и уменьшения поврежденности металла. Вопросы выбора области интегрирования и условий на ее границах, а также влияние расстояния между трещинами и их взаимного расположения друг относительно друга на процессы деформирования и залечивания микродефектов исследовались в [14].

В настоящей работе изучаются процессы изменения введенных параметров залеченности и поврежденности металла от времени и начальной поврежденности при воздействии на него импульсным ВЭМП. Рассматриваются вопросы выбора количества представительных элементов, составляющих область интегрирования, изучается влияние формы микродефектов и расстояния между ними.

\section{2. Электротермомеханическая модель}

Исследование проводится на основе конечно-элементной модели воздействия электромагнитного поля на материал с микродефектами [13-14].

Рассматривается бесконечный поврежденный токопроводящий материал, содержащий одинаковые по форме, размерам и ориентации в пространстве дефекты типа микротрещин, максимальный размер которых по одной из осей значительно меньше максимальных размеров по двум другим осям $h_{0} \ll l_{0}$. Предполагается, что дефекты в материале располагаются упорядоченно в узлах прямоугольной решетки. Представительный элемент объема является прямоугольником (в пространственном случае - параллелепипедом), в центре которого расположена плоская микротрещина. Материал подвергается воздействию короткоимпульсного ВЭМП с плотностью тока от $10^{8}-10^{10} \mathrm{~A} / \mathrm{M}^{2}$ и продолжительностью $10^{-5}-10^{-4}$ с. Считается, что плоскости микротрещин перпендикулярны вектору плотности электрического тока, индуцируемого полем.

В процессе воздействия ВЭМП в материале протекают следующие физические процессы: электромагнитный, механический и тепловой. Характерное время каждого из этих процессов приблизительно обратно пропорционально скорости распространения соответствующих возмущений. Следовательно, время, требуемое для установления электромагнитных и механических процессов, рассматриваемых в настоящей работе, составляет соответственно $10^{-13}-10^{-12}$ с и $10^{-7}-10^{-8}$ с, что существенно меньше времени внешнего воздействия источника тока $\left(10^{-5}-10^{-4} \mathrm{c}\right)$. Поэтому для получения электрического потенциала $\varphi$, в проводящем материале используется закон сохранения заряда (в предположении, что ток в образце является установившемся), выписанный в вариационной постановке [15]. При этом для проводника считается справедливым закон Ома. Поле перемещений $\boldsymbol{u}$ определяется из уравнений равновесия, записанных в форме принципа виртуальной работы [15].

Поле температуры Т определяется из закона сохранения энергии [15]. Поскольку время электромагнитного воздействия на материал мало $\left(10^{-5}-10^{-4} \mathrm{c}\right)$, а градиенты температуры в окрестности вершин трещин очень велики $\left(10^{7 \circ} \mathrm{C} / \mathrm{M}\right)[16]$, то теплопроводностью пренебрегаем и считаем процесс адиабатическим. В получающемся эволюционном уравнении для температуры учитывается тепло, выделяемое в единице объема в текущей конфигурации тела за единицу времени за счёт протекания электрического тока в соответствие с законом ДжоуляЛенца, тепло, выделяемое при пластическом деформировании, а также скрытое тепло, поглощаемое в процессе плавления и испарения. При вычислении поля температуры это уравнение дополняется условиями поглощения скрытого тепла во время перехода вещества из одного 
агрегатного состояния в другое, а именно, при плавлении и испарении соответственно [13].

Деформации предполагаются конечными. Принимается аддитивность скоростей упругих, пластических и температурных деформаций. Для скоростей упругих и пластических деформаций полагается справедливым соответственно закон Гука для изотропного тела и ассоциированный закон течения с условием пластичности Мизеса. Связь между скоростями температурных деформаций и производной температуры полагается линейной.

Температура в рассматриваемых процессах изменяется в диапазоне значений от комнатной до температуры испарения металла [16]. Поэтому в предлагаемой модели все физико-механические характеристики материала (плотность, удельная теплоемкость, электропроводность, коэффициент температурного расширения, упругие модули, предел текучести и т. д.), входящие в вышеприведенные уравнения, зависели от температуры [16-18]. В точках, где материал расплавился, в соответствии с экспериментальными данными [17-18] происходит резкое изменение всех физических свойств материала, а где материал полностью испарился, полагаются нулевыми вектор плотности тока и тензор напряжений, а температура равной температуре испарения.

Задача решается для областей, содержащих один, четыре и шесть представительных элементов (микротрещин). В силу симметрии представительных элементов используемые области интегрирования состояли из половин или четвертей представительных элементов, на горизонтальных и вертикальных границах которых ставились условия отсутствия нормальной компоненты вектора перемещений и касательных компонент тензора напряжений, а также задавался невозмущенный наличием дефекта потенциал. Трещина не проводила ток, поэтому нормальная производная потенциала на ее границе полагалась равной нулю. Поверхность трещины принималась свободной от напряжений. Начальные поля температуры, перемещений и электрического потенциала полагались однородными $\left(T_{0}=20^{\circ} \mathrm{C}, \boldsymbol{u}_{0}=0, \varphi_{0}=0\right)$.

Поскольку изучаются процессы, происходящие на микроскопическом уровне в поликристаллическом металле, то, следуя первым работам теории повреждающихся сред [19], поврежденность ассоциировалась с образованием в материале пустот (пористостью) вследствие образования и развития микротрещин и микропор в процессе нагружения. Обозначим $V(t)$ - объем единичного микродефекта, $V_{0}=V(0)$ - начальный объем дефекта, $V_{\text {re }}$ - объем представительного элемента, в котором находится такой микродефект. Определим параметры поврежденности $f=f(t)$ и залеченности $\chi=\chi(t)$ следующим образом:

$$
f(t)=\frac{V(t)}{V_{\mathrm{re}}}, \quad \chi(t)=\frac{V(0)-V(t)}{V(0)} .
$$

Начальная поврежденность материала в момент времени $t=0$ будет равна $f_{0}=f(0)=V_{0} / V_{\mathrm{re}}$. Поскольку объем микродефекта при воздействии ВЭМП уменьшается [13 - 14], то, следовательно, в результате воздействия полем поврежденность материала будет уменьшаться, а залеченность увеличиваться.

\section{3. Результаты моделирования}

Все вышеописанные уравнения электротермомеханической задачи совместно с граничными, контактными и начальными условиями решались численно. Расчеты проводились для плоской деформации с использованием линейных четырехузловых изопараметрических и трехузловых конечных элементов. Перестройка сетки осуществлялась на основе смешанного эйлеро-лагранжева метода. Температура не является степенью свободы задачи. Эволюционное уравнение для температуры решалось непосредственно в каждой точке интегрирования с использованием аппроксимации производной температуры обратной разностью по времени. Для вычислений использовался пакет программ АСТРА ИПМex РАН.

Моделирование проводилось для образцов из цинка, физико-механические свойства и их зависимости от температуры принимались в соответствие с [17 - 18].

Размеры (длина $a$ и ширина $b$ ) представительных элементов варьировались в диапазоне 15-75 мкм, что обусловлено разбросом размеров зерен поликристаллического цинка.

Рассматриваемые формы микротрещин приведены на (Рис. 1a-1c). Для полноты картины рассматривался гантелевидный микродефект (Рис. 1d). Все дефекты имели одинаковую начальную длину $l_{0}=10$ мкм и одинаковую площадь. Таким образом, начальная поврежденность $f_{0}$ для всех форм микродефектов получалась одинаковой. В расчетах она изменялась в диапазоне $f_{0}=0,27-2,45 \%$.

Разность потенциалов (на единицу длины), используемая в расчетах, составляла $534.34 \mathrm{mB} / \mathrm{MM}$, которой соответствовал ток в материале без дефектов плотностью 8.95 кА/мм². Разность потенциалов принималась постоянной в течение всего времени действия импульса $\tau_{0}=90$ мкс.

В работах [13 - 14] было показано, что при пропускании электрического тока через образец с микротрещинами в их окрестности возникают большие градиенты электрического поля, приводящие к значительному увеличению плотности тока в окрестности вершин трещин по сравнению с плотностью тока, приложенного к образцу. Это вызывает быстрый неоднородный локальный нагрев и плавление материала, сопровождаемые его тепловым расширением. Неоднородное тепловое расши-

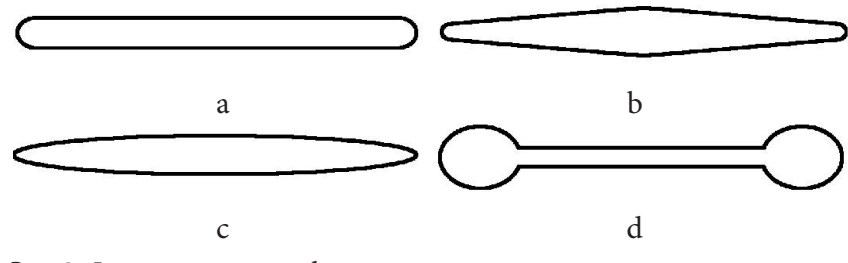

Рис. 1. Формы микродефектов: горизонтальные микротрещины с закругленными вершинами (a); сужающиеся микротрещины c закругленными вершинами (b); эллиптические микротрещины (c); гантелеподобные микродефекты (d).

Fig. 1. Shapes of microdefects: horizontal microcracks with rounded tips (a); narrow microcracks with rounded tips (b); elliptical microcracks (c); dumbbell microdefects (d). 
рение приводит к большим сжимающим напряжениям (давление может составлять свыше $100 \mathrm{MПа)} \mathrm{в} \mathrm{окрест-}$ ности микротрещины и, как следствие, одновременному смыканию берегов (уменьшению ширины), уменьшению длины трещины и выбросу расплавленного материала в трещину (Рис. 2-3). Данный процесс происходит до тех пор, пока струя расплавленного металла не оказывается полностью зажатой берегами трещины, а берега трещины «сваренными» расплавленным металлом (см. рис. 5 работы [13]). Далее весь описанный процесс повторяется.

Расчеты показали, что во всем исследуемом диапазоне расстояний между дефектами (при любой начальной поврежденности) залеченность $\chi(t)$ и поврежденность $f(t)$ при воздействии ВЭМП изменяются во времени одинаково независимо от того рассчитываем мы их в областях интегрирования, состоящих из одного или нескольких представительных элементов. Максимальное отклонение полученных кривых $\chi(t)$ для областей интегрирования с 1,4 и 6 представительными элементами составило 0.09\%. Таким образом, при моделировании процесса залечивания микротрещин можно без потери точности ограничиться рассмотрением в качестве области интегрирования одной четверти представительного элемента (Рис. 2-3), задавая на ее границах вышеописанные краевые условия.

На Рис. 4 приведены зависимости залеченности $\chi$ и поврежденности $f$ от времени $t$ (мкс) для представительных квадратных элементов различных размеров $(a=b)$ с микродефектами в форме горизонтальной, сужающейся и эллиптической микротрещин, а также гантели (Рис. 1a-1d). Во всем исследуемом диапазоне расстояний между микротрещинами кривые изменения залеченности $\chi(t)$ и поврежденности $f(t)$ практически совпадают друг с другом для одинаковой начальной поврежденности $f_{0}$. Этот эффект (независимости $\chi(t)$ и $f(t)$
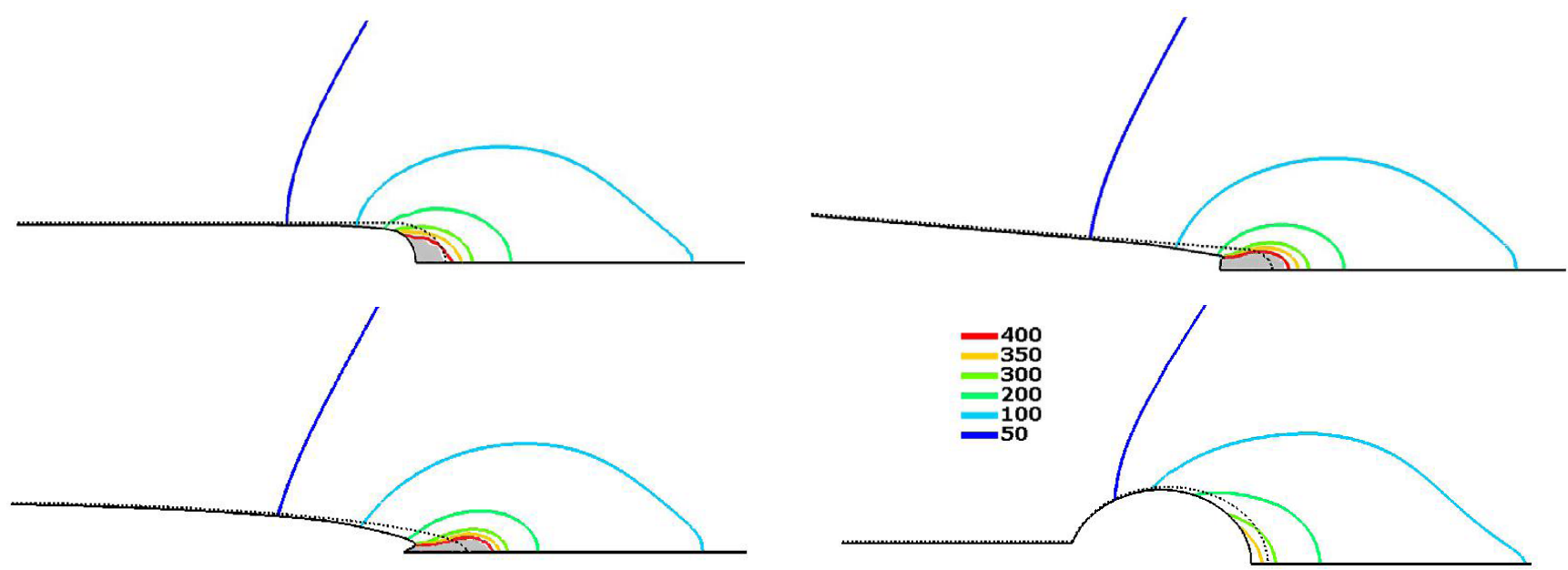

Pис. 2. (Color online) Залечивание различных микродефектов в момент времени $t=25.4$ мкс (штриховой линией показаны первоначальные границы дефектов) и изолинии температуры $\left({ }^{\circ} \mathrm{C}\right)$, серым цветом показана область, где металл расплавился.

Fig. 2. (Color online) Healing of various microdefects at time $t=25.4 \mu \mathrm{s}$ (the dashed line shows the initial boundaries of the defects) and the temperature isolines $\left({ }^{\circ} \mathrm{C}\right)$, the area where the metal melted is shown in gray.
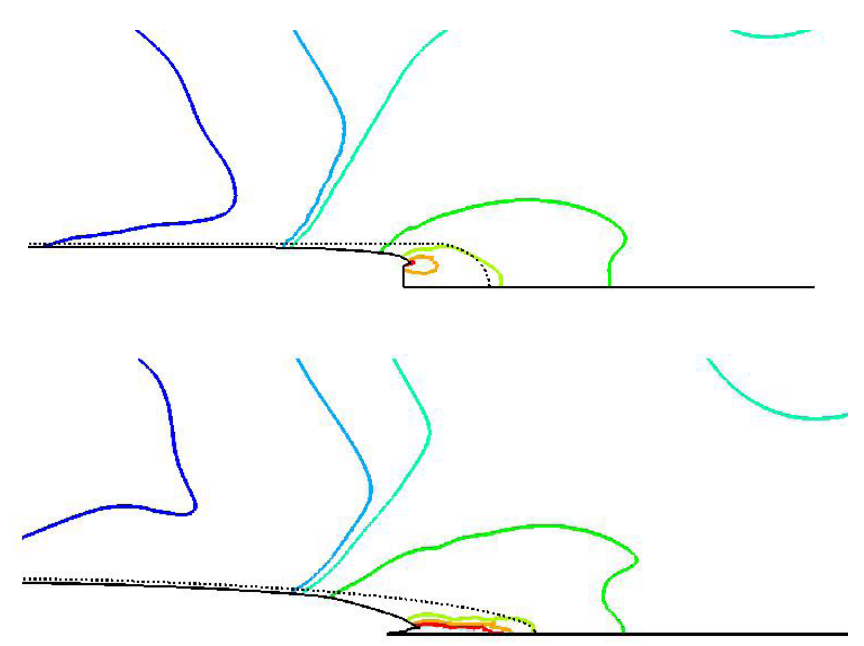
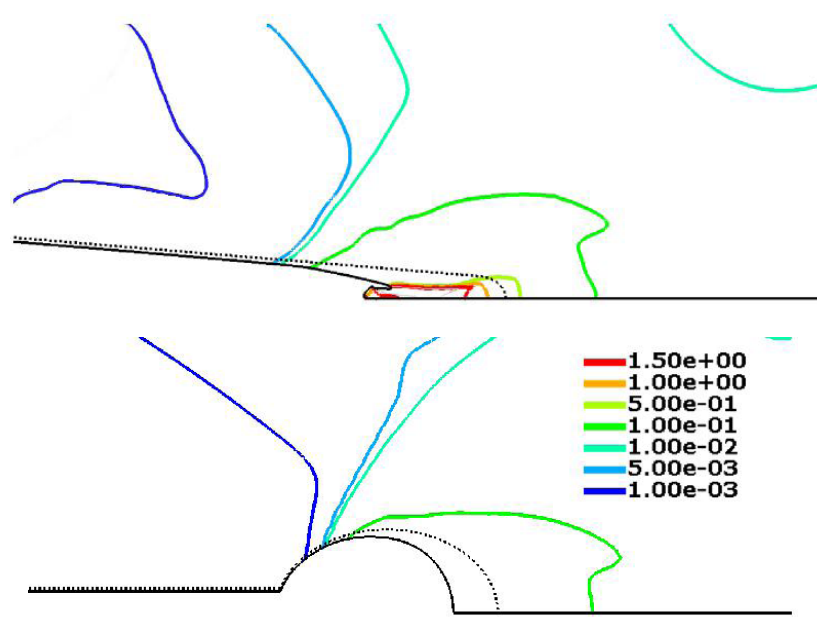

Pис. 3. (Color online) Границы различных микродефектов (штриховой линией показаны первоначальные границы дефектов) в момент времени $t=48.0$ мкс и изолинии второго инварианта тензора пластических деформаций.

Fig. 3. (Color online) The boundaries of various microdefects (the dashed line shows the initial boundaries of the defects) at time $t=48.0 \mu$ s and the isolines of the second invariant of the plastic strains tensor. 

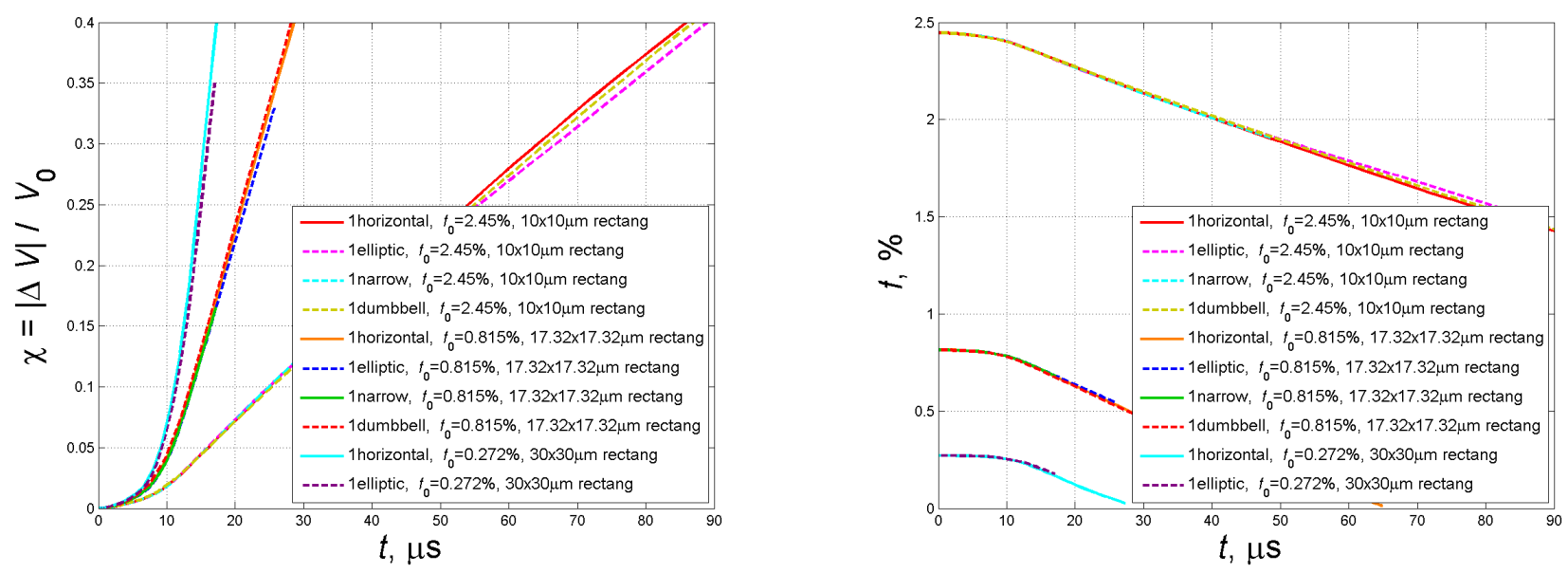

Рис. 4. Зависимости (а) залеченности $\chi$ (b) поврежденности $f$ от времени $t$ (мкс) для микродефектов различных форм (плоская, сужающаяся, эллиптическая микротрещины и гантель) при различной начальной поврежденности $f_{0}$.

Fig. 4. Dependences (a) healed $\chi$ (b) damage $f$ from time $t(\mu \mathrm{s})$ for microdefects of various shapes (horizontal, narrow, elliptical microcracks and dumbbells) for different initial damage $f_{0}$.

от формы микродефекта) имеет место даже для микродефекта, имеющего кардинально иную форму 一 гантель.

Изменение длины и/или ширины представительного элемента $(a \neq b)$ в пределах $25 \%$ от указанной на Рис. 4 (при постоянной $f_{0}$ ) практически не изменяло зависимостей $\chi(t)$ и $f(t)$ (Рис. 4).

Остаточные пластические деформации в окрестности вершин микродефектов достигают сотен процентов (Рис. 3). Это дает основания полагать, что восстановление формы дефекта до первоначальных размеров происходить не будет.

Зависимости, приведенные на Рис. 4b хорошо аппроксимируются линейными функциями:

$$
f(t)=\left\{\begin{array}{cl}
f_{0}, & t<t_{0}, \\
f_{0}-C\left(t-t_{0}\right), & t \geq t_{0},
\end{array}\right.
$$

где коэффициент $C=1,228 \cdot 10^{2} \mathrm{c}^{-1}$, пороговое время $t_{0}=9,63$ мкс. Из (1) и (2) легко получается аппроксимацию зависимости $\chi(t)$ (Рис. $4 \mathrm{a}$ ) из которой следует, что залеченность металла растет со скоростью, обратно пропорциональной начальной поврежденности материала.

Полученные результаты качественно согласуются с имеющимися экспериментами [2,6-10], в частности с тем, что для начала процессов уменьшения поврежденности и увеличения залеченности требуется определенная пороговая энергия [2].

\section{4. Выводы}

Для изменений залеченности и поврежденности материала во времени при воздействии на материал импульсом ВЭМП определяющим является не форма микродефекта (при одинаковой длине микродефекта), а введенный параметр поврежденности материала. Моделирование показывает, что при одинаковой начальной поврежденности материала $f_{0}$, существенные изменения формы микродефектов и расстояний между ними практически не влияют на зависимости $\chi(t)$ и $f(t)$.
Полученные при моделировании зависимости $\chi(t)$ и $f(t)$ с хорошей точностью аппроксимируются кусочно-линейными функциями. Откуда можно заключить, что независимо от начальной поврежденности до некоторого порогового момента времени $t_{0}$ все микротрещины в материале не залечиваются и поврежденность материала не меняется под действием поля. Начиная с момента времени $t_{0}$, все микротрещины начинают залечиваться. При этом поврежденность материала уменьшается с постоянной скоростью, которая опять же не зависит от начальной поврежденности.

Благодарность/Aknowledgements. Работа выполнена при финансовой поддержке РФФИ (гранть №№ 15-08-08693, 18-08-00958)./The investigatin is executed at financial support of RFBR (grants №№ 15-08-08693, 18-0800958).

\section{Литература/References}

1. Beklemishev N. N., Koryagin N.I., Shapiro G.C. Izv. Akad. Nauk SSSR. Metals, 4, 184 (1984). (in Russian) [Беклемишев Н.Н., Корягин Н.И., Шапиро Г.С. Известия АН СССР, Мелаллы. 4, 184 (1984)]

2. Beklemishev N. N., Kukudzhanov V.N., Porokhov V. A. et al. Preprint No. 372, Moscow, IPM AN SSSR. (1989) 56 p. (in Russian) [Беклемишев Н.Н., Кукуджанов В.Н., Порохов В.А. и др. Пластичность и прочность металлических материалов с учетом импульсного воздействия высокоэнергетического электромагнитного поля. Препринт № 372., М. ИПМ АН СССР. 1989. 56 c.]

3. Klyushnikov V.D., Ovchinnikov I. V. Mech. Solids. 23 (4), 113 (1988).

4. Ovchinnikov I. V. J. Problems of Strength. 6, 54 (1993).

5. Kukudzhanov V.N., Kolomiets-Romanenko A. V. Mech. Solids. 45 (3), 465 (2010).

6. Song Hui, Wang Zhong-Jin, Gao Tie-Jun. Trans. 
Nonferrous Soc. China. 17, 87 (2007).

7. Troitskii O.A., Baranov Yu.V., Avraamov Y.S., Shlyapin A.D. Physical fundamentals and technologies of processing advanced materials (theory, technology, structure, and properties). Vol. 1., Moscow-Izhevsk, Inst. Comp.Science, (2004) 590 p. (in Russian) [Троицкий О.А. Баранов Ю.В., Аврамов Ю.С., Шляпин А. Д. Физические основы и технологии обработки современных материалов (теория, технология, структура и свойства). Т. 1. Москва-Ижевск. Инст. комп.иссл. 2004. 590 с.]

8. Conrad H. Final Report ARO Proposal No 23090-MS, ARO Funding Document DAAL03-86-K-0015, U.S. Army Research Office, Raleigh, NC State University. (1989) $52 \mathrm{p}$.

9. Zuev L. B., Tsellermaer V. Ya., Gromov V.E., and Murav'ev V.V. Ultrasonic monitoring of the accumulation of aging damage and recovery of the useful lifetime of industrial parts. Tech. Phys. 49 (2), 1094 (1997).

10. Zuev L. B., Sosnin O. V., Chirakadze D.Z., Gromov V.E., and Murav'ev V.V. J. Appl. Mech. Tech.Phys. 39 (4), 639 (1998).

11. Liu T. J. C. Int. J. Mech., Aerosp., Indust., Mechatr. Manuf. Engineering, 4 (5), 387 (2010)

12. Yu J., Zhang H., Deng D., Hao S., Iqbal A. Chinese J. Mech.
Engineering, 27 (4), 745, (2014).

13. Kukudzhanov K.V. PNRPU Mechanics Bulletin. 4, 138 - 158 (2015) DOI: 10.15593/perm.mech/2015.4.09 (in Russian) [Кукуджанов К.В. Вестник ПНИПУ. Механика. 4, 138 (2015).]

14. Kukudzhanov K.V., Levitin A.L. PNRPU Mechanics Bulletin. 2, 89 (2016) (in Russian) [Кукуджанов К.В., Левитин А.Л. Вестник ПНИПУ. Механика. 2, 89 (2016)]

15. Zienkiewicz O.C., Taylor R.L., Zhu J.Z. The Finite Element Method: Its Basis and Fundamentals. (6ed.), Elsevier. (2005) 613 p.

16. Finkel' V. M., Golovin Yu. I., Sletkov A. A. Sov. Phys. Dokl. 237 (2), 325 - 327 (1977).

17. Gavrilin I. V. Melting and crystallization of metals and alloys. Vladimir, VGU. (2000) 260 p. (in Russian) [Гаврилин И. В. Плавление и кристаллизация металлов и сплавов. Владимир, ВГУ. 2000. 260 с.]

18. Pikunov M.V. Metallurgy of the melts. A course of lectures. Moscow, MISA. (2005) 286 p. (in Russian) [Пикунов М.В. Металлургия расплавов. Курс лекций. М. МИСиС. 2005. 286 с.].

19. Kachanov L. M. Izv. Akad. Nauk SSSR. OTN. 8, 26 (1958) (in Russian) [Качанов Л. М. Известия АН СССР, ОТН. 8, $26(1958)]$ 